\title{
A response to "Competency frameworks: universal or local" by Mortaz Hejri and Jalili (2012)
}

\author{
Anne Mette Morcke · Tim Dornan • Berit Eika
}

Received: 26 June 2013/ Accepted: 27 June 2013

(C) Springer Science+Business Media Dordrecht 2013

First, we would like to thank Hejri and Jalili (Hejri and Jalili 2013) for endorsing the main point in our critical review of outcome (competency) based education (Morcke et al. 2012). We agree with the two points they make: that global prescriptions of curriculum content can be insensitive to cultural differences; and that effective curriculum development calls for local engagement. For the record, the phrase they criticise is not one we wrote, but the conclusion of other authors whose work we reviewed. Their point, nevertheless, is important enough to deserve a reaction. It is one thing to say the CanMEDS competency framework has been extensively adopted around the world. It is another thing to say CanMEDS is equally suited to all cultures. What can be said, however, is that it travels well! A framework, which was developed for use in Canada, has been adopted in Denmark as a framework across pre- and postgraduate training and across all four medical schools, which have different curricula and different approaches to learning. In the Netherlands, likewise, CanMEDS has provided useful as a national organizing framework for competency based postgraduate education. The secret of its success may be that it leaves the scope for local interpretation, which Hejri and Jalili (Hejri and Jalili 2013) ask for. But therein lies a big problem of competency-based education. What is gained in generalizability is lost in specificity.

Our review did not conclude that all medical schools around the world should adopt the same framework. To the contrary, we agree with Hejri and Jalili that medical schools "should evaluate the risks and benefits of developing a new set of outcomes before adopting an existing framework." What we did conclude was that outcome-based

This reply refers to the article available at doi:10.1007/s10459-012-9426-4.

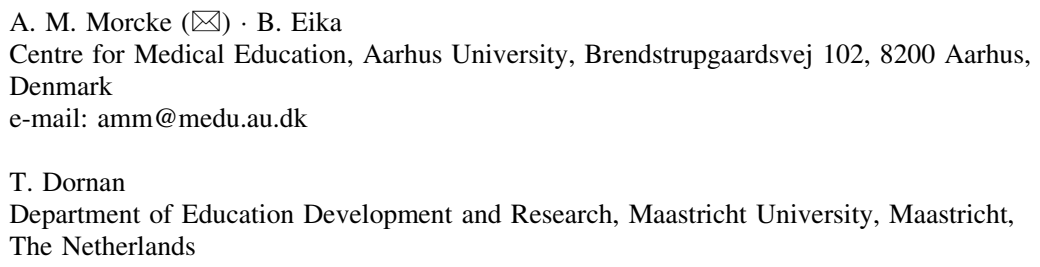


education and competency frameworks can provide a logical basis for blueprinting assessments, particularly assessment of observable behaviors. Their danger is that, at least when inappropriately applied, they can devalue those parts of medical education that are messy and complicated. We particularly call for research that tries to answer the question "how does outcome-based education work, for whom, and in what circumstances?" The medical education research community knows little about what learning outcomes are good for; what the connections are between learning outcomes and teaching; when outcomes are helpful for teachers and when not; when and how they are useful for selfdirected learning. To those questions, Hejri and Jalili (2013) add an important question about how they can be culturally (in)sensitive. We strongly warn against blindly adopting an existing framework in any medical school, including the Tehran University of Medical Sciences. Medical Schools have to draw on theory and local traditions to craft and carefully evaluate their curriculum revisions if the field, as well as the curricula themselves, are to move forward.

\section{References}

Hejri, S. M., \& Jalili, M. (2013). Competency frameworks: Universal or local. Advances in Health Sciences Education (in press).

Morcke, A. M., Dornan, T., \& Eika, B. (2012). Outcome (competency) based education: An exploration of its origins, theoretical basis, and empirical evidence. Advances in Health Sciences Education. doi:10.1007/s10459-012-9405-9. 